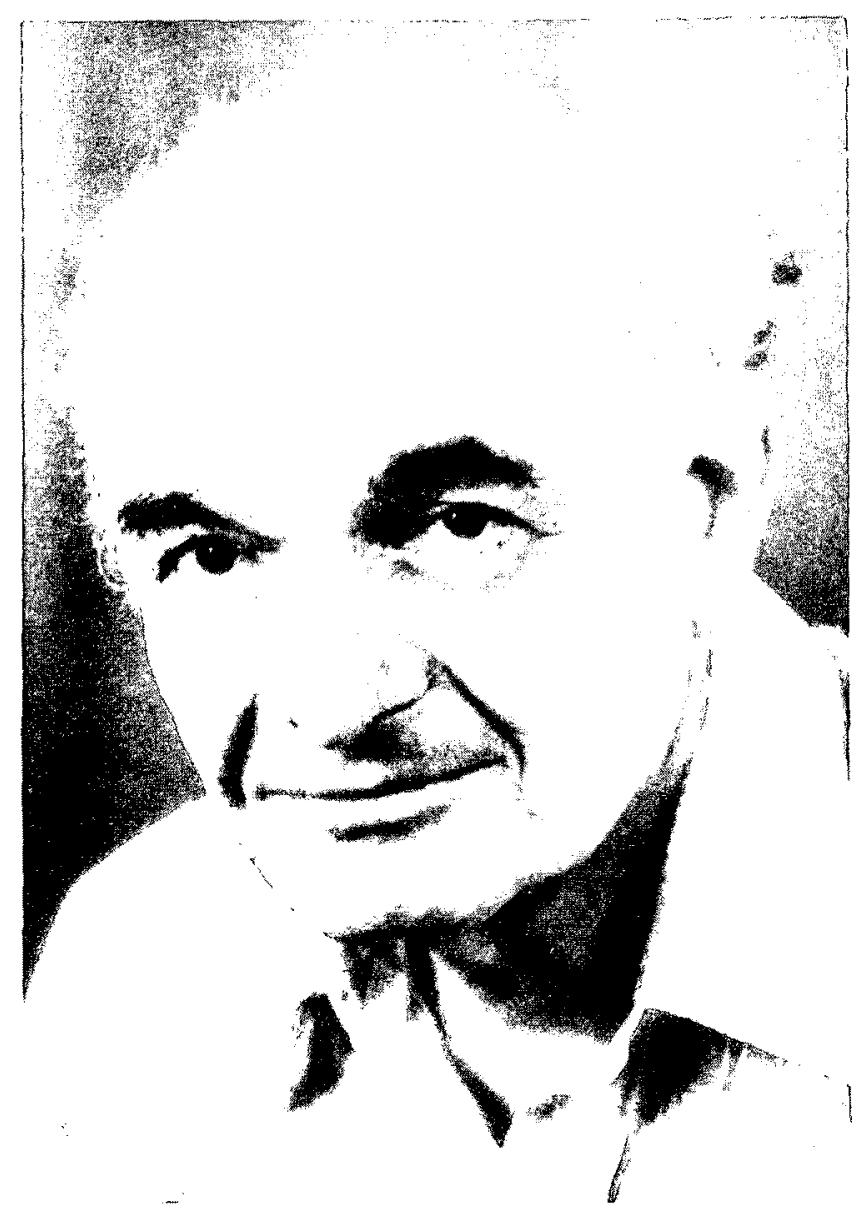

\title{
Louis E. Guttman
}

(1916-1987)

Louis (Eliyahu) Guttman, Scientific Director of The Israel Institute of Applied Social Research (IIASR) and Professor of Social and Psychological Assessment at The Hebrew University of Jerusalem, was born in Brooklyn, New York, on February 10, 1916, and died in Minneapolis, Minnesota, on October 25, 1987, while on a visit to the United States to deliver lectures and to get medical treatment. He was rated among the top 62 ranking world social scientists since the beginning of the 20th century and was well known for his many contributions to multivariate analysis and basic theoretical matters in scientific research and statistics.

Louis Guttman received both his undergraduate (BA, 1936) and graduate training (MA, 1939) at the University of Minnesota, earning his doctorate in social and psychological measurement in 1942. During his long and distinguished career he had multiple affiliations with both governmental and academic institutions. "He was the major force behind conducting a scientifically based job evaluation and classification of the Israel civil service as a basis for reform of its grading system." He was an expert consultant to the US Secretary of War, Washington, D.C. (1941-1945) and had multiple appointments both as 
chairman and consultant to various Israeli agencies (1956-1987) in addition to being the Scientific Director of IIASR, an institute he founded and led for many years since 1947. His academic appointments included Cornell (1941-1950 and again in 1972-1978), Harvard (1954 and 1971, jointly with Massachusettes Institute of Technology), Michigan State University as Distinguished Visiting Professor (1962-1963), The University of Michigan as Visiting Professor of Sociology and Psychology (1964-1965), The University of Texas at Austin as a Visiting Professor of Psychology (1982-1983), The University of Minnesota as a Visiting Professor of Psychology (1987- ), and The Hebrew University of Jerusalem (1955-1987).

Attesting to his wide-ranging interests and abilities he was warmly embraced by sociologists, psychologists and statisticians alike as can be seen from the many different journals in which he had publications and the many society memberships he held both in the United States and in Israel, covering all three of these disciplines, as well as being voted a Foreign Honorary Member of the American Academy of Arts and Sciences.

Awards and honors include his appointment as Fellow, Center for Advanced Study in the Behavioral Sciences (1955-1956), the Rothschild Prize for Social Science (1963), his Andrew White Professorship-at-large from Cornell (1972-1978), the Outstanding Achievement Award from the Regents of the University of Minnesota (1974), The Israel Prize in the Social Sciences (1978), the Educational Testing Service Measurement Award from Princeton (1984), and his election as President of the Psychometric Society (1970).

Louis Guttman published in numerous journals and books covering a half-century from 1938, both as a sole author or in collaboration with others, some $300+$ pages appearing in Psychometrika alone. Many of his earlier papers are still quoted in the literature as being relevant to current statistical and mathematical advances. To summarize all of this material would be a herculean task, so we have divided Guttman's publications into three main areas: factor analysis, scaling, and theoretical, covering what each of the contributors deems to be most significant.

Louis Guttman (according to Ledyard Tucker) left us a tremendous legacy of major developments in the theory and practice of factor analysis. We are truly indebted to him for his many outstanding contributions. Serious students, theoreticians and practitioners of factor analysis, must have studied these developments in detail and must take them into account in further developments and analyses. This author always found it a great learning experience to study Guttman's publications. His elegant mathematical treatments are both thorough and imaginative. His philosophic points demand close study and evaluation to properly fathom their meaning. In the few words to follow we can not review Guttman's developments in detail; rather, we will review the nature of some of his developments along with theoretical implications and their impact on factor analytic practice.

A number of Guttman's best known publications involved the linear algebra of factor analysis, frequently in relation to linear multiple regression. Several of these contributions were influenced by his views of the structure of human abilities and attitudes, which will be expanded on in subsequent paragraphs. Guttman's early publication (1940) on "Multiple Rectilinear Prediction and the Resolution into Components" presented some very interesting and important relations, including a formula for the inverse of a correlation matrix when this matrix could be represented by common factors and uniqueness. This formula involved inverting no matrix larger than the number of common factors and has been found to be extremely useful in many contexts. A second highly important publication (1944), "General Theory and Methods for Matric Factoring", presented a general form for most methods of factor extraction from a covariance or correlation matrix. He followed this paper with (1952), "Multiple Group Methods for Common-Factor Analysis: 
Their Basis, Computation, and Interpretation", which gave the theoretic basis for factor extraction methods proposed by others. These methods provide quick, easy factor analyses which involve nothing more than scratch paper, possibly supplemented by a handheld calculator. The results should be considered as nothing more than an approximate factor analysis since they do not depend on all correlations and may ignore factors not represented by groups of variates.

Several of Guttman's contributions have been incorporated into computer packages for calculating factors. First is his work on the number of common factors. Lower bounds for the number of common factors were presented in his article (1954), "Some Necessary Conditions for Common-Factor Analysis". Guttman argued that more factors should be extracted than in the usual practice of removing as few factors as necessary to leave small residual correlations. He presented three lower bounds for the number of common factors, the weakest one which underestimated the number of factors most is used in many computer programs. This lower bound is the number of eigenvalues of the correlation matrix which equal or exceed unity. These packages ignore Guttman's inequality and may lead to less than desirable results. Individuals using these computer programs for data analysis should be aware that too few factors may have been extracted yielding somewhat confused results. This article was followed by a work on communality estimation (1956), "Best Possible' Systematic Estimates of Communalities", in which he recommended the use of SMC's as first estimates, each SMC being the squared multiple correlation of a variate with all other variates in the battery. Present practice follows this recommendation. Further discussion of these issues are given in (1957), "Simple Proofs of Relations Between the Communality Problem and Multiple Correlation" and (1958), "To What Extent can Communalities Reduce Rank?".

Guttman's study of the internal properties of factor analysis using linear multiple regression theory led to his development of "Image Theory for the Structure of Quantitative Variates" (1953). Scores on each variate are decomposed into a component, $x_{j}^{*}$, predicted from all other variates in a battery, the image scores. Anti-image scores, $e_{j}$, are the errors of estimate. Guttman showed important properties of the covariance matrix of image scores and that of anti-image scores. These properties have led other individuals to the use of image matrices in factor analytic procedures. Guttman followed up with "The Matrices of Linear Least Squares Image Analysis" (1960).

Scores on factors in common-factor analysis was a continuing concern to Guttman. This concern led him to his highly important paper "The Determinancy of Factor Score Matrices With Implications for Five Other Basic Problems of Common-Factor Theory" (1955). He showed, for a population of individuals, that factor score matrices could be computed - in contrast to the common belief that they could be only estimated. A most important point stated by Guttman, however, was that many such factor score matrices could be computed: the factor scores were not unique. The commonly used factor score estimated matrices are not among the factor score matrices which satisfy the basic common-factor equation. Many users of factor analysis appear to be uninformed about Guttman's development and use factor score estimates as if these were the theoretic factor scores. Greater care in the conceptualization and use of factor analysis should be followed by these many users of common-factor analysis-as pointed out by Guttman.

Guttman's major developments in scale analysis were related to factor analysis in a number of ways. First was his introduction of the principal components of scale analysis in his initial work on scale construction, "The Quantification of a Class of Attributes: A Theory and Method of Scale Construction", (1941). As Guttman indicated, these principal components of scale analysis are closely related to principal components in factor analysis. The scale analysis scores and weights are quite analogous to principal component 
scores and weights. He derived the principal components of scale analysis to maximize internal consistency in the sense of least squares for responses to perfectly scalable items. In subsequent book chapters, "The Principal Components of Scale Analysis", (1950) and, "The Principal Components of Scalable Attitudes", (1954), he suggested interpretations for these principal components in their order of size: metric properties, intensity, closure, and involution. Factor analyses at the item level produce what has been called difficulty factors, which appear to be closely related to Guttman's principal components of scalable items.

A second major relation between scale analysis and factor analysis was Guttman's use of his technique for smallest space analysis (later named similarity structure analysis) for a configuration of points for investigating the structure of the interrelations among intelligence tests. In this development Guttman used a function of the correlations between tests as interpoint distances and obtained a geometric representation having a minimum number of dimensions to represent the correlations.

Guttman's radex formed a basic element in his theoretical view of the structure of intelligence, abilities, and attitudes. He described "A New Approach to Factor Analysis: The Radex", (1954) in which variables were laid out in a theoretical and geometric configuration involving simplexes and circumplexes. Differences in kind of ability were laid out in a circular fashion, while differences in degree were represented by differences in distance from an origin. For tests having the same level of complexity the representation was a circle having a given radius, thus: they formed a circumplex. Tests of one kind differing in complexity formed a simplex, represented by a ray from the origin. Levels of correlations were reflected by distances between points in this configuration. He pointed out that measures taken in a time sequence during learning would form a simplex with measures closer in time correlating more highly than measures more distant in time. A number of bodies of data follow this pattern leading others to follow up on this observation. Guttman further described the simplex in his paper, "A Generalized Simplex for Factor Analysis" (1955). He also searched results from published studies for evidence of circumplex structures, "Empirical Verification of the Radex Structure of Mental Abilities and Personality Traits" (1957).

Guttman discussed his general theoretical position on factor analysis in his presentation at a symposium on the future of factor analysis (1958). A general structure of intelligence and abilities could be conceived in terms of facets of a Cartesian product of these sets: $I$ for intellectual abilities, $C$ for types of content, and $T$ for types of things or objects. For a given value of any of these sets, the other two sets could be considered as forming a radex with each element giving rise to a factor. Thus, his view was that there were many factors. This was in agreement with his view that the common practice of extracting as few factors as would yield small residuals was incorrect. He disagreed with the view that there was a small number of common factors. His theoretic structure led to questions concerning other aspects of common factor analysis, such as the communality problem. Guttman argued that this theoretic structure provided a more useful conception of the variety of factors of intelligence and abilities as well as for personality traits.

Professor Tucker concludes by saying: "We value greatly Louis Guttman's outstanding contributions to the theory and practice of factor analysis. His developments were characterized by exceptional insight into problems and originality in solutions. $\mathrm{He}$ showed superior logical and mathematical sophistication in his publications. These contributions have had and should have a lasting impact on the theory and practice of factor analysis. Guttman truly was a leader and one of the great people in this field."

From yet another perspective, that of a former student, Samuel Shye evaluates Louis Guttman's influence on him and expands on Tucker's remarks regarding Guttman's 
scaling contributions and his theoretical orientation. As was often his wont, Guttman would present a symmetric matrix of coefficients to a generally "sophisticated" audience and ask them, "What is the most salient feature of this empirically observed matrix?" After receiving many ingenious responses, completely missing the obvious, he would point out that all the interrelations were positive. In other contexts he would present similar problems, illustrating his typical emphasis on grasping the essentials of a complex situation first, before proceeding to more esoteric issues.

Uniformity of sign in a matrix was associated by Guttman with a common range for the observed items. Items have a common range if a single substantive concept can rank the set of possible responses to each of them. The attempt to identify correspondences between a conceptual aspect of observational items and an empirical aspect of the observations, is central to Guttman's scientific contribution. Indeed, he has defined scientific theory as:

An hypothesis of a correspondence between a definitional system for a universe of observations and an aspect of the empirical structure of those observations, together with a rationale for such an hypothesis.

Items whose range is ordered from very positive to very negative by the concept of "behavior towards an object" were defined as attitude items in (1982 paper with Shlomit Levy), paving the way to the "First Law of Attitude":

If any two items are selected from the universe of attitude items towards a given object, and if the population observed is not selected artificially, then the population regressions between these two items will be monotone and with positive or zero sign.

A similar "First Law of Intelligence" was formulated for intelligence items, defined as those with a range from "very right" to "very wrong" with respect to an objective rule.

These laws summarized phenomena that had been observed by psychologists for some time, namely, that correlations between mental tests (and between attitudes) almost always turn out to be positive. Guttman's contribution here is twofold: First, the recognition that these observations present an opportunity to state laws, as such; and second, the attempt to state a formal definition for intelligence items (not just "intelligence") and for attitude items (not just "attitude"), without which, of course, no such laws can be formulated. The value of stating laws for scientific development can be great indeed: a law summarizes past findings and tells researchers what to normally expect, that is, what is the incumbent (null) hypothesis. On the other hand, it presents a challenge for future investigations: "Under what circumstances does the law not hold?" or "How can it be further refined, for example, beyond mere correlation signs?"

It is indeed to the latter question, that of structural laws, that much of Guttman's efforts have been directed in recent years, and for which he has advanced facet theory or, more correctly, facet metatheory. If the concept common to all item ranges (responses to be recorded) determines the boundaries of the content universe delineated by the items, concepts that classify the item domain (questions asked) determine the internal structure of that universe. Every concept by which the question part of an item set may be classified, was termed a domain facet. For example, the facet "behavior modality" may classify items according to whether they concern the cognitive, affective, or instrumental kinds of behavior. The facets of "life area" may classify items according to whether they ask about housing, work, leisure, health, family life, and so forth. Of course, any number of domain facets may classify a given item set. In the words of Clyde Coombs: "Guttman's facet design ... is the only substantial attempt to provide a general theory for characterizing 
domain." In addition to the book edited in 1985 by David Canter on facet theory, which Louis was fond of promoting at every opportunity, a former student, now at Indiana University, L. Suzanne Dancer, is producing yet another book on this same subject.

In multidimensional scaling or, as he preferred to call it, multidimensional similarity structure analysis (SSA), Guttman saw a simple procedure capable of transcending the limitations of factor analysis, that is, one that permits testing of hypothesis formulated in terms of domain facets. For here we have a straightforward visual representation of what he called the content universe. If this physical representation of the item space could be systematically partitioned into regions in accordance with the domain facets, a correspondence would be established between an aspect of the conceptual-definitional framework (facet) and an aspect of the empirical structure (partition) of the observations.

In a unidimensional item space, all items lie on a line, named a simplex. A twodimensional item space may be found to be partitionable into stripes, concentric circles, or circularly ordered sections, giving rise to patterns termed duplex, radex, and so on. In three-dimensional item spaces, a spherex, cylindrex, and other configurations may be observed. The essential thing to note, however, is that all these patterns are definable only on the basis of substantive classifications or domain facets. Thus, Guttman has proposed the radex, and later the cylindrex, of intelligence items; the radex of "well-being" (which he has regarded as a variety of attitude); and, so on.

Guttman advocated the integration of data analysis and research contents and was critical of works which focused on data condensation where little regard was paid to potential lawfulness in substantive content. He was also critical of routine applications of statistical inference when statistical significance replaced scientific significance. Indeed, Louis had a wealth of illustrations of common statistical errors made by social scientists, which he summarized in an important paper, "What is Not What in Statistics" (1977). He was also a strong advocate of replication as a check on one's hypotheses. These were some of the important principles he tried to inculcate in his students and others in the scientific community. In this respect, Guttman's views were remarkably like those of Thurstone, the great psychometrician who had probably influenced him more than any other scholar.

Guttman expressed his disappointment with the way the concept of a cumulative "Guttman scale" for appraising individuals was understood and particularly by what he regarded as the antiscientific activity of constructing such scales in his 1971 presidential address to the Psychometric Society (mea culpa-J. C. L.). The scalability of a given item set, selected by an a priori content criterion, is an hypothesis which may or may not be confirmed. More often it is not and the methods of multiple scaling, for example, partial order scalogram analysis (POSA), or multidimensional scalogram analysis (MSA), later named multidimensional structure analysis, are then necessary to distinguish among individuals by their observed profiles.

One of the technical problems that fascinated Louis Guttman most recently was that of characterizing relationships between the item space (which structures the content universe) and the profile space (which structures the population). He had made a number of observations relating SSA spaces to POSA spaces and had suggested that the Guttman scale, a unidimensional profile space, corresponds to a point, a null-dimensional item space. Unfortunately, he was unable to pursue this line of reasoning further (as was the case with a number of other unfinished conjectures which he had filed away to be worked on later).

Shye concludes his remarks with: "Louis" death is a great loss to the social and behavioral science world, but the great contributions he has made to the field provide an inspiration, indeed a research strategy, for his many students and colleagues."

As for myself, (J. C. L.), I am most profoundly indebted to Louis Guttman, who over 
these past 25 years has guided, inspired, and motivated me to develop and expand upon his many innovations in the scaling area. In recognition of his mentorship I have published a book, The Guttman-Lingoes Nonmetric Program Series (1973), to include his name in the title, even though a number of these programs were co-developed with others, for example, Coombs, Roskam, and Borg. These programs have a world-wide distribution and we owe it largely to Guttman for presenting a well-thought-out and clear formulation of the problems to be solved for generating the set of error-free algorithms embodied in these computer programs. After completing the very first version of SSA, Louis was anxious to run some data he was to present at the 1964 ETS conference on testing problems. The results were so striking that he told me that he was going to abandon his originally intended presentation and use the SSA results to illustrate the multifaceted nature of intelligence tests and its obvious radex structure. In one way this illustrates how a theory (facet theory) led to a method (SSA), which in turn advanced theory-not too different from the role often played by instrumentation in the physical sciences. In addition to SSA (using the rank-image principle) he had a rather unique formulation for mathematically defining spatial contiguity in MSA-I, which was one of the first programs we worked on after SSA-I. Here he employed another technique for insuring convergence based on the absolute value principle. The initial configuration for this program dated back to his early work on qualitative data (1941). The two papers which best give some historical perspective on these developments are his letter to John Ross (1967) and his presidential address for the Psychometric Society (1971) plus a 1978 commemorative volume by Shye.

Louis was a complex man: strong in conviction, mild but dominant in personality; kindly toward his family and friends; tolerant and patient, but would not "suffer fools gladly"; argumentative sometimes to the point of arrogance; relativistic and absolutist; a man who strongly believed in basics and the scientific tradition; he was above all a man of impeccable scientific and mathematical integrity. His ideas inspired others to expand on his methods (sometimes, however, without his commitment to the rationale for developing the procedures in the first instance, e.g., as in the case of the "Guttman scale"). To him procedures were but a means to an end-the scientific enterprise always assumed for him primacy. If he alienated others by his insistence on the principles he so deeply believed in, for example, on the question of statistical versus scientific significance, it was not based on any meaness of spirit, but was well-intentioned and he was certainly not the only one in either the social science or the statistical community at large who called "Fire!" and, in my opinion, justifiably so. Nevertheless, after seeing his prospectus for "An Introduction to Facet Theory and Data Analysis", my belated wish is that he had expended less energy on "fighting" such demons when it could have been more profitably spent on the book. What a loss! When all is said and done, however, a "giant" will not be remembered for his height alone, but for the progeny he bore in the form of stimulating others-and, that, he has certainly done!

The members of the Psychometric Society wish to offer our heartfelt condolences and sympathy to his wife, Ruth, his three children, Adi, Nurit, and Daphna, and his six grandchildren.

University of Michigan

JAMES C. LINGOES

University of Illinois

LEDYARD R. TUCKER

Israel Institute of

Applied Social Research

Samuel Shye

Psychometrika will posthumously publish an article by Louis Guttman entitled "Eta, Disco, Odisco, and $F "$ in the September 1988 issue. 